\title{
Free generators in free inverse semigroups
}

\author{
N.R. Reilly
}

\begin{abstract}
Using the characterization of the free inverse semigroup $F$ on a set $X$, given by Scheiblich, a necessary and sufficient condition is found for a subset $K$ of an inverse semigroup $S$ to be a set of free generators for the inverse subsemigroup of $S$ generated by $K$. It is then shown that any non-idempotent element of $F$ generates the free inverse semigroup on one generator and that if $|X|>2$ then $F$ contains the free inverse semigroup on a countable number of generators. In addition, it is shown that if $|X|=1$ then $E$ does not contain the free inverse semigroup on two generators.
\end{abstract}

Let $X$ be a non-empty set. By a free inverse semigroup on $X$ is meant an ordered pair $(F, f)$ where $F$ is an inverse semigroup and $f$ is a mapping of $X$ into $F$ such that for any mapping $g$ of $X$ into an inverse semigroup $S$ there is a unique homomorphism $h$ of $F$ into $S$ with $g=f \circ h$. The inverse semigroup $F$ is then unique to within isomorphism and is referred to as the free inverse semigroup on $X$. In [5], Šăn establishes that inverse semigroups form a variety, from which it follows that free inverse semigroups exist. Alternative approaches are discussed by Eberhart and Selden [2] and McAlister [3].

In his recent paper [6], Scheiblich has given a valuable characterization of the free inverse semigroup on $X$ in terms of partial transformations of the power set of the non-identity elements in the free group on $X$. We begin by describing this characterization. Basic

Received 27 June 1972. 
information about inverse semigroups and unexplained notation will be found in [1].

Let $X$ be a non-empty set and $X^{\prime}$ be a set disjoint from $X$ and in one-to-one correspondence with $X$. Let this correspondence be denoted by $x \leftrightarrow x^{-1}$. We also write $x=\left(x^{-1}\right)^{-1}$.

A word in $Y=X \cup X^{\prime}$ is any finite sequence, $a_{1} \ldots a_{n}$, of elements from $Y$ and a reduced word is any finite sequence, $a_{1} \ldots a_{n}$, of elements from $Y$ such that $a_{i} \neq a_{i+1}^{-1}, i=1, \ldots, n-1$. The empty sequence, denoted by 1 , is also a reduced word. Let $G$ denote the set of reduced words and $R=G \backslash\{1\}$. For any elements $g=a_{1} \ldots a_{m}$, $h=b_{1} \cdots b_{n}$ of $\vec{G}$ let $g h$ denote the reduced word obtained from $a_{1} \ldots a_{m} b_{1} \ldots b_{n}$ by removing any adjacent pairs $y y^{-1}, y \in Y$ successively until a reduced word is obtained. This defines a binary operation on $G$ with respect to which $G$ is a group - the free group on $X$. In particular, for any $x \in X$, the elements $x$ and $x^{-1}$ (as sequences in $G$ ) are group inverses.

For any $w=a_{1} \ldots a_{n} \in R$, let

$$
I(w)=\left\{a_{1}, a_{1} a_{2}, \ldots, a_{1} \ldots a_{n}\right\},
$$

that is, let $I(w)$ denote the set of initial segments of $w$.

Let $E=\{A \subseteq R: A \neq \varnothing, A$ is finite and $I(w) \subseteq A$ for all $w \in A\}$. Thus $E$ is the set of subsets of $R$ which are closed with respect to taking initial segments. If $A, B \in E$ then $A \cup B \in E$. Thus, if $E$ is partially ordered by defining $A \leq B$ if $A \supseteq B$, then $E$ is a semilattice such that $A \cup B$ is the greatest lower bound of $A$ and $B$. For any $A \in E$ we write $A^{1}$ for $A \cup\{1\}$.

For each $x \in X$, define a permutation $x p$ of $R$ as follows: for $w \in R$, 


$$
w(x p)= \begin{cases}x^{-1} & \text { if } w=x, \\ x^{-1} w & \text { otherwise. }\end{cases}
$$

The mapping $\rho: x \rightarrow x \rho$ extends to a homomorphism (in fact, an isomorphism) $\rho$ of $G$ into the group, $P(R)$, of permutations of $R$. It is convenient to note here that for any $w \in R, x \in X$,

$$
w\left(x^{-1} \rho\right)= \begin{cases}x & \text { if } w=x^{-1} \\ x w & \text { otherwise. }\end{cases}
$$

This homomorphism induces another homomorphism, which we also denote by $\rho$, of $G$ into $P(E)$, defined as follows: for any $A \in E, g \in G$,

$$
A(g \rho)=\{a(g \rho): \alpha \in A\} \text {. }
$$

Let $F=\left\{(A, w) \in E \times G: w \in A^{l}\right\}$ and define a binary operation on $F$ as follows:

$$
(A, w)(B, v)=\left(A \cup B(w p)^{-1}, w v\right) \text {. }
$$

Define a mapping $f: X \rightarrow F$ by $x f=(\{x\}, x)$.

THEOREM 1.1 ([6], Scheiblich). With respect to the operation defined in (1), $F$ is an inverse semigrow and $(F, f)$ is the free inverse semigroup on $X$.

For any element $U=(A, a)$ in $F$, let $\Delta^{\prime}(U)=A$.

The following lemma lists some simple observations from [6] which are used later without further comment.

LEMMA 1.2. Let $W=(A, a)$ and $w_{i}=\left(A_{i}, a_{i}\right), i=1, \ldots, n$ be any elements of $F$.

1. $W$ is an idempotent in $F$ if and only if $a=1$.

2. $W^{-1}=\left(A(\alpha \rho), a^{-1}\right)$.

3. $W W^{-1}=(A, 1), W^{-1} W=(A(a p), 1)$ and $\Delta^{\prime}(W)=A=\Delta^{\prime}\left(W W^{-1}\right)$. 


$$
\begin{aligned}
& \Delta^{\prime}\left(\prod_{i=1}^{n} w_{i}\right)=A_{1} \cup A_{2}\left(a_{1} \rho\right)^{-1} \cup A_{3}\left(a_{2} \rho\right)^{-1}\left(a_{1} \rho\right)^{-1} \cup \ldots u \\
& A_{n}\left(a_{n-1} \rho\right)^{-1} \ldots\left(a_{1} \rho\right)^{-1} .
\end{aligned}
$$

There are some immediate observations that can be made regarding $F$. For instance, since every idempotent is of the form $(A, 1)$ with $A \in E$ there are $2|x|$ maximal idempotents in $F$, where $|X|$ denotes the cardinality of $X$. Also, the following observations can be made regarding Green's relations $H, L, R, D$ and $J$.

LEMMA 1.3. Let $U=(A, a), V=(B, b)$ be any elements of $F$.

(1) $U R V \Leftrightarrow A=B$.

(2) $U L V \Leftrightarrow A(a p)=B(b \rho)$.

(3) $U H V \Leftrightarrow A=B$ and $a=b$.

Thus $H$ is the identity relation.

(4) $\quad$ UOV $\Leftrightarrow A\left(a^{\prime} \rho\right)=B\left(b^{\prime} \rho\right)$ for some $a^{\prime} \in A^{I}, b^{\prime} \in B^{1}$.

(5) $J=0$.

(6) $\left|R_{U}\right|=|A|+3=\left|L_{U}\right|$.

(7) $\left|D_{U}\right|=(|A|+1)^{2}$.

Proof. Parts (1), (2), (3), (6) and (7) are immediate consequences of Lemma 1.2.

For part (4), first suppose that $U D V$. Then, for some $W=(C, c)$ we must have $U R W$ and $W L V$. Then $A=C$ and $C(c \rho)=B(b \rho)$. Thus $A(c \rho)=B(b \rho)$ where $c \in C^{l}=A^{l}, b \in B^{l}$.

Conversely, let $A\left(a^{\prime} \rho\right)=B\left(b^{\prime} \rho\right)$ for some $a^{\prime} \in A^{l}, b^{\prime} \in B^{l}$. Let $W=\left(A, a^{\prime}\right), Z=\left(B, b^{\prime}\right)$. Then $U R W, W L Z, Z R V$. Hence UDV.

One always has $0 \subseteq J$ and so, for part (5), we wish to establish the converse inclusion. So let $U J V$. Then, since $V \in F U F$, there exist elements $W=(C, c), Z=(D, d)$ such that $V=W U Z$. Hence $b=c a d$ 
and

$$
\begin{aligned}
B=\Delta^{\prime}(V) & =\Delta^{\prime}(W U Z) \\
& =C \cup A(c \rho)^{-1} \cup D(\alpha \rho)^{-1}(c \rho)^{-1} .
\end{aligned}
$$

Hence, $A(c \rho)^{-1} \subseteq B$ and $A \subseteq B(c \rho)$. Similarly, since $U \in F V F$, there exists a $c^{\prime} \in G$ such that $B \subseteq A\left(c^{\prime} \rho\right)$. Since $A$ and $B$ are finite, since $A$ and $A\left(c^{\prime} \rho\right)$ have the same cardinality and since $B$ and $B(c \rho)$ have the same cardinality we must have $A=B(c p)$. Thus $A(a \rho)=B(c a \rho)=B\left(b d^{-1} \rho\right)$. Let $d=d_{1} \cdots d_{n}$. Since $d \in D$ we have $d_{1} \in \nu$. If $b d^{-1} \in I(b)$ then we have the required result. Otherwise $b d^{-1}=u d_{1}^{-1}$ where either $u=1$ or $u d_{1}^{-1}$ is in reduced form. Then $d_{1}\left(d b^{-1}\right) \rho \in D\left(d b^{-1} \rho\right)=D(c a \rho)^{-1} \subseteq B$. But

$$
d_{1}\left(d b^{-1}\right) \rho=d_{1}\left(d_{1} u^{-1} \rho\right)=u d_{1}^{-1}=b d^{-1} \text {. }
$$

Thus $A(a \rho)=B\left(b d^{-1} \rho\right)$ where $b d^{-1} \in B, a \in A$. Thus UDV.

\section{Free generators}

For any subset $K$ of an inverse semigroup $S$ let $\langle K\rangle$ denote the inverse subsemigroup of $S$ generated by $K$. If $K=\{U\}$, for some $U \in S$ then we write $\langle K\rangle=\langle U\rangle$. For any subset $K$ of a group $H$ we denote by $\langle K\rangle$ the subgroup of $H$ generated by $K$. Let $K \subseteq S$, and let $i$ denote the embedding of $K$ into $S$. If $(\langle K\rangle, i)$ is the free inverse semigroup on $K$ then we say that $K$ is a set of free generators for $\langle K\rangle$. For any subset $k$ of $S, k^{-1}=\left\{k^{-1}: k \in K\right\}$. For any elements $a_{1}, \ldots, a_{n}$ in $s$ we write $\prod_{i=1}^{n} a_{i}=a_{1} a_{2} \ldots a_{n}$.

We first observe that the sets which are sets of free generators for $F$ are very restricted.

PROPOSITION 2.1. Let $W \subseteq F$ and $\langle W\rangle=F$. Then $W$ is a set of free generators for $F$ if and only if, $W \subset X f \cup(X f)^{-1}$ and, for each 
$x \in X, \quad\left|\operatorname{Wn}\left(x f,(x f)^{-1}\right\}\right|=1$.

Proof. It is clear that if the condition is satisfied then $W$ is a set of free generators. So suppose that $W$ is a set of free generators.

We first show that $W \cup W^{-1}=(X f) \cup(X f)^{-1}$. Let $x \in X$. Then, for some $w_{1}, \ldots, w_{n} \in W \cup W^{-1}$,

$$
(\{x\}, x)=w_{1} \cdots w_{n} .
$$

Hence

$$
\begin{aligned}
\{x\}^{\prime} & =\Delta^{\prime}\left(w_{1} \ldots w_{n}\right) \\
& =\Delta^{\prime}\left(w_{1}\right) \cup \ldots .
\end{aligned}
$$

Thus $\Delta^{\prime}\left(W_{1}\right)=\{x\}$ and so $W_{1}=(\{x\}, x)=x f$ or $W_{1}=(\{x\}, 1)$. In the latter case, $W_{1}$ is an idempotent which is clearly impossible, since $W$ is a set of free generators. Hence $x f=W_{1} \in W \cup W^{-1}$. Therefore $(X f) \cup(X f)^{-1} \subseteq W \cap W^{-1}$. Since both $X f$ and $W$ are sets of free free generators for $F$, we must have $(X f) \cup(X f)^{-1}=W \cup W^{-1}$. Thus, for any $x \in X$, we must have $\left\{x f,(x f)^{-1}\right\} \cap W \neq \emptyset$. But since $W$ is a set of free generators we cannot have $\left\{x f,(x f)^{-1}\right\} \subseteq W$. Hence we have the desired conclusion.

We now give a general criterion for a subset $K$ of an inverse semigroup $S$ to be a set of free generators for $\langle K\rangle$.

THEOREM 2.2. Let $K$ be a subset of an inverse semigroup $S$. Then $K$ is a set of free generators for $\langle K\rangle$ if and only if the following condition is satisfied:

(K) If $Y \in K \cup K^{-1}$ and $Y Y^{-1} \geq F_{1} \cdots F_{n}$ where $F_{j}=Y_{j 1} \ldots Y_{j n(j)^{y}(j)} y_{j}^{-1} Y_{j 1}^{-1}$ for some $y_{j k} \in K \cup K^{-1}$ such that $Y_{j k} \neq Y_{j k+1}^{-1}$ for $k=1, \ldots, n(j)-1$, $j=1, \ldots, n$, then $Y=Y_{j 1}$ for some $j$. 
Free inverse semigroups

413

Proof. Let $\theta: X \rightarrow K$ be a bijection of some set $X$ onto $K$. Let $(F, f)$ be the free inverse semigroup on $X$ as described in Section 1 . Then $\theta$ determines a unique epimorphism of $E$ onto $\langle K\}$, which we also denote by $\theta$. Clearly $K$ is a set of free generators for $\langle K\rangle$ if and only if $\theta$ is an isomorphism.

First suppose then that $\theta$ is an isomorphism. We wish to show that condition $(K)$ is satisfied. Let $Y$ be some element of $K \cup K^{-1}$ such that

$$
Y Y^{-1} \geq F_{1} \ldots F_{n}
$$

where, for $j=1, \ldots, n$,

$$
F_{j}=Y_{j l} \ldots Y_{j n(j)^{Y}}^{-1} \ldots Y_{j 1}^{-1}
$$

for some elements $Y_{j k} \in K \cup K^{-1}$ such that $Y_{j k} \neq Y_{j k+1}^{-1}$, for any $j=1, \ldots, n, k=1, \ldots, n(j)$. Here $n(j)$ denotes some integer that depends on $j$.

Let $U, U_{j k}$ be elements of $X f \cup(X f)^{-1}$ such that

$$
y \theta^{-1}=U=(\{y\}, y)
$$

and

$$
Y_{j k} \theta^{-1}=U_{j k}=\left(\left\{_{j k}\right\}, y_{j k}\right)
$$

for some $y, y_{j k} \in X \cup X^{\prime}$. Let $E_{j}=F_{j} \theta^{-1}, j=1, \ldots, n$. Then

$$
U U^{-1} \geq E_{1} \ldots E_{n} \text {. }
$$

Hence

$$
\begin{aligned}
\Delta^{\prime}(U) & =\Delta^{\prime}\left(U U^{-1}\right) \subseteq \Delta^{\prime}\left(E_{1} \ldots E_{n}\right) \\
& =\bigcup_{j=1}^{n} \Delta^{\prime}\left(E_{j}\right) \\
& =\bigcup_{j=1}^{n} \Delta^{\prime}\left(U_{j 1} \ldots U_{j n(j)}\right) .
\end{aligned}
$$

https://doi.org/10.1017/S0004972700045251 Published online by Cambridge University Press 
Therefore, $y \in \Delta^{\prime}\left(U_{j l} \ldots U_{j n(j)}\right)$, for some $j$. Now, since $y_{j k} \neq Y_{j k+1}^{-1}$, for any $j, k$, we have that $U_{j k} \neq U_{j k+1}^{-1}$ and hence that $y_{j k} \neq y_{j k+1}^{-1}$. Hence the elements $y_{j 1}, y_{j 1} y_{j 2}, \ldots, y_{j 1} y_{j 2} \cdots y_{j n(j)}$ are all in reduced form and so, by Lemma 1.2 (4),

$$
\begin{aligned}
\Delta^{\prime}\left(U_{j 1} \ldots U_{j n}(j)\right. & =\Delta^{\prime}\left(U_{j 1}\right) \cup \Delta^{\prime}\left(U_{j 2}\right)\left(y_{j 1} \rho\right)^{-1} u \ldots \\
& =\left\{y_{j 1}, y_{j 1} y_{j 2}, \ldots, y_{j 1} y_{j 2} \ldots y_{j n(j)}\right\} .
\end{aligned}
$$

Thus the only element of $\Delta^{\prime}\left(U_{j 1} \ldots U_{j n(j)}\right)$ of length one in reduced form is $y_{j l}$. Hence $y=y_{j l}, U=U_{j l}$ and $y=y_{j l}$. Thus $(K)$ is satisfied.

Now suppose that $(K)$ is satisfied. We wish to show that $\theta$ is an isomorphism. Since $\theta$ is clearly an epimorphism, we need only show that $\theta$ is a monomorphism. To this end, we need the following result due to Munn [4].

LEMMA 2.3. Let $T$ be an inverse semigroup and $\tau$ be a congruence on $T$. Then $\tau \subset H$ if and only if $\tau$ is idempotent separating (that is, $(a, b) \in \tau, a^{2}=a$ and $b^{2}=b$ imply that $\left.a=b\right)$.

If we can show that for any distinct idempotents $M, N$ of $F$, $M \theta \neq N \theta$ then the congruence $\theta \circ \theta^{-1}$ induced by $\theta$ is idempotent separating. By Lemma 2.3 this means that $\theta \circ \theta^{-1} \subseteq H$. But, by Lemma 1.3, $H$ is the identity relation. Consequently $\theta$ must be a monomorphism.

So let $M, N$ be distinct idempotents of $F$ such that $M \theta=N \theta$. Then $\Delta^{\prime}(M) \neq \Delta^{\prime}(N)$. Without loss of generality, let $z_{1} \ldots z_{n} \in \Delta^{\prime}(M) \backslash \Delta^{\prime}(N)$, where $n \geq 1, z_{1}, \ldots, z_{n} \in X \cup X^{\prime}$ and $z_{\alpha} \neq z_{\alpha+1}^{-1}, \alpha=1, \ldots, n-1$. Let $z_{i}=\left(\left\{z_{i}\right\}, z_{i}\right), \quad i=1, \ldots, n$. Since $z_{1} z_{2} \cdots z_{n} \in \Delta^{\prime}(M)$, 


$$
\begin{aligned}
\Delta^{\prime}\left(z_{1} z_{2} \ldots z_{n} z_{n}^{-1} \ldots z_{1}^{-1}\right) & =\Delta^{\prime}\left(z_{1} \ldots z_{n}\right) \\
& =\left\{z_{1}, z_{1} z_{2}, \ldots, z_{1} z_{2} \ldots z_{n}\right\} \subseteq \Delta^{\prime}(M),
\end{aligned}
$$

and so

$$
M \leq z_{1} \ldots z_{n} Z_{n}^{-1} \ldots z_{1}^{-1}
$$

Hence,

$$
N \theta=m \theta \leq z_{1} \theta \ldots z_{n}^{\theta \theta z_{n}^{-1} \theta} \ldots z_{1}^{-1} \theta .
$$

Let $\Delta^{\prime}(N)=\left\{n_{1}, \ldots, n_{r}\right\}$, for some integer $r$ and, for $i=1, \ldots, r$, let $n_{i}=v_{i 1} \ldots v_{i k(i)}$ for some $v_{i \alpha} \in X \cup X^{\prime}$ with $v_{i \alpha} \neq v_{i \alpha+1}^{-1}, \alpha=1, \ldots, k(i)-1$. Let $v_{i j}=\left(\left\{v_{i j}\right\}, v_{i j}\right)$, $i=1, \ldots, r, j=1, \ldots, k(i)$. For each $i$, let $B_{i}=I\left(n_{i}\right)$ and $N_{i}=\left(B_{i}, 1\right)$. Then

$$
\begin{aligned}
\Delta^{\prime}(N) & =\bigcup\left\{B_{i}: i=1, \ldots, r\right\}, \\
N & =\prod_{i=1}^{r} N_{i}
\end{aligned}
$$

and

$$
N_{i}=V_{i 1} \cdots V_{i n(i)} V_{i n(i)}^{-1} \cdots V_{i I}^{-1} .
$$

We will show by induction that, for $s=1, \ldots, n$ there exists an $i$ with $z_{1}=v_{i 1}, \ldots, z_{s}=v_{i s}$.

First let $s=1$. Since $z_{1} \theta z_{1}^{-1} \theta \geq z_{1} \theta \ldots z_{n} \theta z_{n}^{-1} \theta \ldots z_{1}^{-1} \theta$, we have that $Z_{1} \theta Z_{1}^{-1} \theta \geq N \theta$. Let $Z_{i}{ }^{\theta}=Y_{i}$ and $V_{i j}{ }^{\theta}=Y_{i j}$. Then $Y_{1} y_{1}^{-1} \geq \prod_{i=1}^{r}\left(y_{i 1} \ldots Y_{i 1}^{-1}\right)$. Hence, by $(K), Y_{1}=y_{i 1}$, for some $i$.

Since $\theta$ is one-to-one on $x f \cup(x f)^{-1}, z_{1}=V_{i I}$. Now suppose that $z_{1}=v_{i 1}, \ldots, z_{s-1}=v_{i s-1}$, for $i=1, \ldots, k$, but not for 
$i=k+1, \ldots, r$. Then $y_{1}=y_{i 1}, \ldots, y_{8-1}=y_{i s-1}$, for $i=1, \ldots, k$, but not for $i=k+1, \ldots, r$. For the sake of brevity, we shall sometimes write $A$ for $Y_{1} \ldots Y_{8-1}$ in what follows.

We have, from (2), that

$$
\begin{aligned}
y_{1} \ldots y_{8} y_{8}^{-1} \ldots y_{1}^{-1} & \geq y_{1} \ldots y_{n}^{y_{n}^{-1}} \ldots y_{1}^{-1} \\
& \geq N \theta .
\end{aligned}
$$

Hence

$$
A^{-1}{ }_{Y_{\theta}} Y_{s}^{-1} A^{-1} A \geq A^{-1}(N \theta) A=\prod_{i=1}^{r} A^{-1}\left(N_{i} \theta\right) A \text {. }
$$

Thus, since $Y_{8} Y_{8}^{-1} \geq A^{-1} A Y_{8} Y_{s}^{-1} A^{-1} A$, we have

$$
y_{s} Y_{S}^{-1} \geq \prod_{i=1}^{r} A^{-1}\left(N_{i} \theta\right) A \text {. }
$$

If $i \leq k$, then

$$
\begin{aligned}
A^{-1}\left(N_{i} \theta\right) A & =A^{-1}\left(A_{i s} \ldots Y_{i n(i)^{Y}}^{Y_{i n(i)}^{-1}} \ldots Y_{i s}^{-1} A^{-1}\right) A \\
& =A^{-1}{ }_{i s} \ldots Y_{i n(i)^{Y}}{ }_{i n(i)}^{-1} \ldots Y_{i s}^{-1} .
\end{aligned}
$$

If $k<i$, then for some integer $p$, dependent upon $i$, such that $0 \leq p<8-1$, we have

$$
\begin{aligned}
& \text { (5) } A^{-1}\left(N_{i}, \theta\right) A=A^{-1} Y_{1} \ldots y_{p}^{Y}{ }_{i p+1} \ldots y_{i n(i)} y_{i n(i)}^{-1} \ldots y_{i p+1}^{-1} y_{p}^{-1} \ldots Y_{1}^{-1} A \\
& =A^{-1} Y_{s-1}^{-1} \ldots Y_{p+1}^{-1} Y_{i p+1} \cdots Y_{i n(i)^{Y}}^{Y_{i n(i)}^{-1}} \cdots \\
& \ldots y_{i p+1}^{-1}{ }_{p+1} \ldots y_{s-1}
\end{aligned}
$$

where $y_{p+1} \neq Y_{i p+1}$. From (3), (4), (5) and condition (K) we must have $Y_{s}=Y_{8-1}^{-1}$ or $Y_{i s}$ (for some $i=1, \ldots, k$ ). But $Y_{s}=Y_{s-1}^{-1}$ implies that $z_{s}=z_{s-1}^{-1}$, a contradiction. Hence $Y_{s}=Y_{i s}$, for some $i=1, \ldots, k$. Hence, for some $i, y_{1}=y_{i 1}, \ldots, y_{s}=Y_{i s}$, and 
$z_{1}=v_{i 1}, \ldots, z_{s}=v_{i s}$. Hence, for some $i, z_{1}=v_{i 1}, \ldots, z_{n}=v_{i n}$, and

$$
z_{1} \cdots z_{n} z_{n}^{-1} \ldots z_{1}^{-1} \geq N_{i} \geq N
$$

Therefore $z_{1} z_{2} \cdots z_{n} \in \Delta^{\prime}(N)$, which contradicts the choice of $z_{1} \cdots z_{n}$. Hence $\theta \circ \theta^{-1}$ is idempotent separating and $\theta$ is an isomorphism.

If, in Theorem 2.2, $S$ is actually the free inverse semigroup $F$ on $X$ as described in Section 1, then we can give a reformulation of the condition (K) which is less cumbersome to apply. In $F, Y Y^{-1} \geq F_{1} \ldots F_{n}$ if and only if $\Delta^{\prime}\left(Y_{Y}^{-1}\right) \subseteq \Delta^{\prime}\left(F_{1} \ldots F_{n}\right)=\bigcup_{j=1}^{n} \Delta^{\prime}\left(F_{j}\right)$. Also $\Delta^{\prime}\left(Y Y^{-1}\right)=\Delta^{\prime}(Y)$ and $\Delta^{\prime}\left(F_{j}\right)=\Delta^{\prime}\left(Y_{j 1} \ldots Y_{j n(j)}\right)$. Thus, in $F$, condition $(K)$ could have been stated as

(K') If $Y \in K \cup K^{\prime}$ and $\Delta^{\prime}(Y) \subseteq \bigcup_{j=1}^{n} \Delta^{\prime}\left(Y_{j I} \ldots Y_{j n(j)}\right)$ for some $Y_{j k} \in K \cup K^{-1}$ such that $Y_{j k} \neq Y_{j k+1}^{-1}$ for $k=1, \ldots, n(j)-1, j=1, \ldots, n$, then $y=y_{j 1}$, for some $j$.

If we take $|K|=1$ in Theorem 2.2, then we obtain the following simple criterion for an element in an inverse semigroup to generate the free inverse semigroup on one generator.

COROLLARY 2.4. Let $U$ be an element of the inverse semigroup $S$. Then $\langle U\rangle$ is the free inverse serrigroup on a single generator if and only if $U U^{-1} \neq U^{-m} U^{m}$ and $U^{-1} U \neq U^{n} U^{-n}$, for any positive integers $m, n$.

This corollary could also be obtained from the characterization of the $\theta$-classes on a free inverse semigroup with one generator due to Eberhart and Seldon [2].

Once again let $F$ denote the free inverse semigroup on $X$. 
COROLLARY 2.5. Let $U$ be any non-idempotent of $F$. Then $U$ is a free generator of $\langle U\rangle$.

Proof. Let $U=(A, u)$ where $u \neq 1$. For any $v \in R$, we define a function $d(v,-): R \rightarrow N$, where $N$ is the set of non-negative integers; for any $c \in R, d(v, c)$ is the largest integer $n$ such that $c=v^{n} c^{\prime}$ for some $c^{\prime} \in G$, where $v_{c}^{n}$ is in reduced form, but $c \neq v^{n+1} c^{\prime \prime}$ in reduced form. Then $d(v, c) \geq 0$, for all $v, c \in R$.

Let $a \in A$ be such that $d(u, a)$ is maximal in $\{d(u, b): b \in A\}$. Since $u \in A, d(u, a) \geq 1$. Clearly $d(u, a)>d(u, b)$, for any $b \in A(u \rho)$, and so $d(u, a)>\max \{d(u, b): b \in A(u \rho)\}$. Also $\max \left\{d(u, b): b \in A(u p)^{p}\right\} \geq \max \left\{d(u, b): b \in A(u p)^{q}\right\}$ for any positive integers $p, q$ with $l \leq p \leq q$. Hence, for any integer $m \geq 1$, $d(u, a)>\max \left\{d(u, b): b \in A(u \rho)^{p}, p=1, \ldots, m\right\}$. Thus $a \vDash A(u \rho) \cup A(u \rho)^{2} \cup \ldots \cup A(u \rho)^{m}=\Delta^{\prime}\left(U^{-m}\right)=\Delta^{\prime}\left(U^{-m} U^{m}\right)$. Therefore, $U U^{-1} \neq U^{-m} U^{m}$, for any integer $m \geq 1$. Similarly, $U^{-1} U \neq U^{n} U^{-n}$, for any integer $n \geq 1$. By Corollary 2.4, we have the desired result.

Let $\gamma: F \rightarrow G$ be such that $(A, u) \gamma=u$. Then clearly $\gamma$ is an epimorphism, $G$ is the maximal group homomorphic image of $F$, and $\gamma \circ \gamma^{-1}$ is the minimum group congruence. It follows easily that if $K=\left\{\left(A_{i}, w_{i}\right): i \in I\right\}$ is a set of free generators for $\langle K\rangle$ then $W=\left\{w_{i}: i \in I\right\}$ is a set of free generators for $\langle W\rangle$.

If $W=\left\{w_{i}: i \in I\right\}$ is a subset of $G$ and a set of free generators for $\langle W\rangle$, it is tempting to conjecture that $K=\left\{\left(I\left(w_{i}\right), w_{i}\right): i \in I\right\}$ will be a set of free generators for $\langle K\rangle$. In general, this will not be the case. Theorem 2.2 can be tailored to this situation as follows.

PROPOSITION 2.6. Let $W=\left\{w_{i}: i \in I\right\}$ be a non-empty subset of $G$ with $W$ disjoint from $W^{-1}$. Let $K=\left\{W_{i}=\left(I\left(w_{i}\right), w_{i}\right): i \in I\right\}$. Then $K$ is a set of free generators for $\langle K\rangle$ if and only if the following condition $\left(K_{1}\right)$ is satisfied. 
$\left(\mathrm{K}_{1}\right)$ For any subset $u_{1}, \ldots, u_{k+1}$ of $W \cup W^{-1}$ with

$$
\begin{aligned}
& u_{\alpha} \neq u_{\alpha+1}^{-1}, \alpha=1, \ldots, k-1, \text { if } u_{k} \ldots u_{1} \in I\left(U_{k+1}\right) \\
& \text { then } u_{k}=u_{k+1} .
\end{aligned}
$$

Proof. First suppose that $K$ is a set of free generators. Let $u_{1}, \ldots, u_{k+1}$ be elements of $w \cup W^{-1}$ such that $u_{\alpha} \neq u_{\alpha+1}^{-1}$, $\alpha=1, \ldots, k-1$, and $u_{k} \ldots u_{1} \in I\left(u_{k+1}\right)$ while $u_{k} \neq u_{k+1}$. Let $k$ be the smallest positive integer for which there are such elements $u_{1}, \ldots, u_{k+1}$.

Let $U_{i}=\left(I\left(u_{i}\right), u_{i}\right), i=1, \ldots, k+1$. Then $U_{i}^{-1}=\left(I\left(u_{i}^{-1}\right), u_{i}^{-1}\right)$.

First suppose that $k=1$. Then $u_{1} \in I\left(u_{2}\right)$ and so $\Delta^{\prime}\left(U_{1}\right) \subseteq \Delta^{\prime}\left(U_{2}\right)$ and, by $\left(K^{\prime}\right), U_{1}=U_{2}$ so that $u_{1}=u_{2}$.

Now suppose that $k \geq 2$. If there is a $j$ such that $2 \leq j \leq k$ and $u_{j-1} \cdots u_{1} \in I\left(u_{j}^{-1}\right)$ then this would contradict the choice of $k$ and the $u_{\alpha}$. Hence

$$
u_{k} \ldots u_{1}=u_{1}\left(u_{2} \rho\right)^{-1} \ldots\left(u_{k} \rho\right)^{-1}
$$

Thus

$$
\begin{aligned}
u_{1} & \in I\left(u_{k+1}\right)\left(u_{k} \rho\right) \ldots\left(u_{2} \rho\right) \\
& =\Delta^{\prime}\left(U_{k+1}\right)\left(u_{k} \rho\right) \ldots\left(u_{2} \rho\right) \\
& \leq \Delta^{\prime}\left(U_{2}^{-1} \ldots \vec{u}_{k}^{-1} U_{k+1}\right)
\end{aligned}
$$

and, by $\left(K^{\prime}\right)$, since $U_{k+1} \neq U_{k}, U_{1}=U_{2}^{-1}$ which is again a contradiction. Hence condition $\left(K_{l}\right)$ must be satisfied.

Now suppose that condition $\left(K_{1}\right)$ is satisfied. Let $y \in K \cup K^{-1}$ and $Y Y^{-1} \geq F_{1} \ldots F_{n}$ where $F_{j}=Y_{j 1} \ldots Y_{j n(j)}{ }_{j n(j)}^{y_{j n}^{-1}} \ldots Y_{j 1}^{-1}$ for some 
$y_{j k} \in K \cup K^{-1}$ such that $y_{j k} \neq Y_{j k+1}^{-1}, k=1, \ldots, n(j)-1$.

Let $y=(I(y), y)$, for some $y \in W \cup W^{-1}$. Then $\Delta^{\prime}\left(Y Y^{-1}\right)=\Delta^{\prime}(Y)=I(y)$. Hence $y \in \Delta^{\prime}\left(F_{j}\right)$, for some $j$, and so $Y Y^{-1} \geq E_{j}$, for some $j$. For convenience, let

$$
F_{j}=F=Y_{1} \ldots Y_{n}^{Y_{n}^{-1}} \ldots Y_{1}^{-1}
$$

where $y_{i}=\left(I\left(y_{i}\right), y_{i}\right), y_{i} \in W \cup W^{-1}$. Then

$$
y \in \Delta^{\prime}\left(y_{1}\right) \cup \Delta^{\prime}\left(y_{2}\right)\left(y_{1} \rho\right)^{-1} \cup \ldots u \Delta^{\prime}\left(y_{n}\right)\left(y_{n-1} \rho\right)^{-1} \ldots\left(y_{1} \rho\right)^{-1} \text {. }
$$

If $y \vDash \Delta^{\prime}\left(y_{1}\right)$, let $r$ be the least positive integer such that

$$
y \in \Delta^{\prime}\left(y_{x}\right)\left(y_{x-1} \rho\right)^{-1} \ldots\left(y_{1} \rho\right)^{-1}
$$

Then $r \geq 2$ and

$$
y\left(y_{1} \rho\right) \ldots\left(y_{r-1} \rho\right) \in \Delta^{\prime}\left(y_{r}\right)
$$

If, for any integer $j$ such that $1 \leq j<r-1$, we have

$$
y\left(y_{1} \rho\right) \ldots\left(y_{j} \rho\right) \in I\left(y_{j+1}\right)=\Delta^{\prime}\left(y_{j+1}\right)
$$

then

$$
y \in \Delta^{\prime}\left(y_{j+1}\right)\left(y_{j} \rho\right)^{-1} \ldots\left(y_{1} \rho\right)^{-1},
$$

contradicting the choice of $r$. Hence

$$
y_{r-1}^{-1} \cdots y_{1}^{-1} y=y\left(y_{1} \rho\right) \ldots\left(y_{r-1} \rho\right) \in \Delta^{\prime}\left(y_{r}\right)=I\left(y_{p}\right)
$$

and, by $\left(k_{1}\right), y_{r-1}^{-1}=y_{r}$ which is a contradiction.

Hence $y \in \Delta^{\prime}\left(y_{1}\right)=I\left(y_{1}\right)$ and, by $\left(k_{1}\right), y=y_{1}$ and $Y=y_{1}$, as required.

Let $W$ be a subset of $G$ satisfying the conditions of Proposition 2.4. If $w_{1}, \ldots, w_{n} \in W \cup W^{-1}$ are such that $w_{i} \neq w_{i+1}^{-1}, i=1, \ldots, n$ 
then it is clear from $\left(\mathrm{K}_{2}\right)$ that $w_{1} \ldots w_{n} \neq 1$. Hence $W$ is a set of free generators for $(W)$. Thus for condition $\left(K_{1}\right)$ to hold it is necessary for $W$ to be a set of free generators of $(W)$. To see that this is not sufficient, consider the case where $X=\left\{x_{1}, x_{2}\right\}$ and $W=\left\{x_{1}, x_{1} x_{2}\right\}$. Then $W$ is a set of free generators for $G$ but, since $x_{1} \in I\left(x_{1} x_{2}\right)$, the set $K=\left\{\left(\left\{x_{1}\right\}, x_{1}\right),\left(\left\{x_{1}, x_{1} x_{2}\right\}, x_{1} x_{2}\right)\right\}$ is not a set of free generators for $\langle K\rangle$.

We use Proposition 2.6 to show that the free inverse semigroup on two generators contains the free inverse semigroup on a countable number of generators.

COROLLARY 2.7. Let $X=\{a, b\}$. Then there is a countable subset $K$ of $F$ such that $K$ is a set of free generators for $\langle K\rangle$.

Proof. For each positive integer $m$, let

$$
w_{m}=a^{m} b^{-1} a b a^{-m-1}
$$

and $K=\left\{\left(I\left(w_{m}\right), w_{m}\right): m=1,2, \ldots\right\}$. It is well known [7] that $W=\left\{w_{m}: m=1,2, \ldots\right\}$ is a subset of a set of free generators of the derived group of $G$. We show that $W$ satisfies condition $\left(K_{1}\right)$.

First we note that the $(m+2)$ nd term in the reduced expression by which $w_{m}$ is defined and the $(m+3)$ rd term in each $w_{m}^{-1}$ (that is, the middle " $a$ " or " $a^{-1 ")}$ is a significant factor in the sense that the reduced form of any expression of the form

$$
u_{k} \ldots u_{1}
$$

where $u_{\alpha} \in W \cup W^{-1}$ and $u_{\alpha} \neq u_{\alpha+1}^{-1}, \alpha=1, \ldots, k-1$, will contain the significant factor of each of $u_{k}, \ldots, u_{1}$.

Now suppose that, for some $u_{1}, \ldots, u_{k+1} \in W \cup w^{-1}$,

$$
u_{k} \cdots u_{1} \in I\left(u_{k+1}\right)
$$


where $u_{\alpha} \neq u_{\alpha+1}^{-1}, \alpha=1, \ldots, k-1$. Let $u_{k}=w_{p} \in W$. Then the reduced form of $u_{k} \ldots u_{1}$ has an initial segment equal to $d_{b}{ }^{-1} a$. Thus

$$
u_{k} \cdots u_{1}=a^{p_{b}-1} a v
$$

for some $v \leqslant G$. Now, $u_{k+1}$ will have an initial segment equal to $a_{b} b_{a}$ if and only if $u_{k+1}=w_{p}$. Hence, $u_{k+1}=w_{p}=u_{k}$, as required.

The case where $u_{k} \in W^{-1}$ is treated similarly. Thus condition $\left(k_{1}\right)$ is satisfied.

Finally, we show that any two non-idempotent elements of the free inverse semigroup on a single generator will not be free generators of the inverse subsemigroup that they generate.

PROPOSITION 2.8. Let $X=\{x\}$. Let $U, V$ be any two elements in $F$. Then $\{U, V\}$ is not a set of free generators for $H=\langle\{U, V\}\rangle$.

Proof. Since the result is immediate if either $U$ or $V$ is an idempotent, we assume that neither is an idempotent. Clearly $U$ and $V$ are free generators for $H$ if and only if $U^{E}$ and $V^{\delta}$ are free generators, for any $\varepsilon, \delta \in\{1,-1\}$. Thus we can assume that $U=\left(A, x^{m}\right), \quad V=\left(B, x^{n}\right)$ where $m$ and $n$ are positive integers. For any non-zero integers $p, q$ with $p>q$ we shall. write $\left[x^{p}, x^{q}\right]= \begin{cases}{\left[x^{p}, x^{p-1}, \ldots, x^{q+1}, x^{q}\right]} & \text { if either } p, q>0 \text { or } \\ & p, q<0, \\ {\left[x^{p}, x^{p-1}, \ldots, x, x^{-1}, \ldots, x^{q}\right]} & \text { if } q<0<p .\end{cases}$ Then $A=\left[x^{a}, x^{b}\right]$ and $B=\left[x^{c}, x^{d}\right]$, for some non-zero integers $a, b, c, d$ with $a \geq m, b$ and $c \geq n, d$. Also $b, c \leq 1$. Now 


$$
A\left(x^{n} \rho\right)= \begin{cases}{\left[x^{l-n}, x^{b-n}\right]} & \text { if } b<0<a-n, \\ {\left[x^{a-n}, x^{b-n-1}\right]} & \text { if } 0<a-n, b=1, \\ {\left[x^{a-n-1}, x^{b-n}\right]} & \text { if } a-n \leq 0 \text { and } b<0, \\ {\left[x^{a-n-1}, x^{b-n-1}\right]} & \text { if } a-n \leq 0, b=1 .\end{cases}
$$

We shall only carry through the argument for one of these cases since the remaining cases may be treated similarly. Let $a-n \leq 0, b<0$. Then

$$
\begin{aligned}
A\left(x^{n} \rho\right)\left(x^{-m} \rho\right) & =\left[x^{a-n-1}, x^{b-n}\right]\left(x^{-m} \rho\right) \\
& =\left\{\begin{array}{lll}
{\left[x^{a-n-1+m}, x^{b-n+m}\right]} & \text { if } a-n-1+m<0, \\
{\left[x^{a-n+m}, x^{b-n+m}\right]} & \text { if } b-n+m<0 \leq a-n-1+m, \\
{\left[x^{a-n+m}, x^{b-n+m+1}\right]} & \text { if } 0 \leq b-n+m .
\end{array}\right.
\end{aligned}
$$

Since $b<0$ and $m \leq a$, we have $b-n+m+1 \leq a-n$. Thus, for $a-n \leq 0$, $b<0$ we have

$$
A\left(x^{n} \rho\right) \cup A\left(x^{n} \rho\right)\left(x^{-m_{\rho}}\right)=\left\{\begin{array}{ll}
{\left[x^{a-n+m}, x^{b-n}\right]} & \text { if } a-n-1+m \geq 0, \\
{\left[x^{a-n+m-1}, x^{b-n}\right]} & \text { if } a-n-1+m<0 .
\end{array} .\right.
$$

Hence, for sufficiently large $r$,

$$
A\left(x^{n} \rho\right) \cup A\left(x^{n} \rho\right)\left(x^{-m} \rho\right) \cup \ldots \cup A\left(x^{n} \rho\right)\left(x^{-m} \rho\right)^{r}=\left[x^{a-n+1 m}, x^{b-n}\right] .
$$

Let this set be denoted by $J$. Then, for sufficiently large $r$,

$$
\begin{aligned}
& \Delta^{\prime}\left(v^{-1} v^{r}\right)=\Delta^{\prime}\left(v^{-1}\right) \cup J \\
& \supseteq \begin{cases}{\left[x^{-1}, x^{d-n}\right] \cup J,} & \text { if either } n \neq 1 \text { or } d \neq 1, \\
\left\{x^{-1}\right\} \cup J, & \text { if } n=d=1\end{cases} \\
& \supseteq\left[x^{a-n+r m}, x^{d}\right] \text {. }
\end{aligned}
$$

In particular, if $r$ is such that $a-n+r m>c$, then

$$
\Delta^{\prime}\left(V^{-1} U^{n}\right) \supseteq\left[x^{c}, x^{d}\right]=\Delta^{\prime}(V) \text {. }
$$

Since $V \neq V^{-1}$, condition $(K)$ is not satisfied. All other possible 
orderings of $a, b, m$ and $n$ produce the same conclusion and so $\{U, V\}$ is not a set of free generators for $\langle\{U, V\}\rangle$.

In conclusion, we observe that if $K=\{U, V\}$ where $U=\left(\left\{x, x^{2}\right\}, x\right), \quad V=\left(\left\{x^{-1}, x^{-2}\right\}, x^{-1}\right)$ then $\langle K\rangle$ contains three maximal idempotents, namely, $U U^{-1}=\left(\left\{x, x^{2}\right\}, 1\right)$, $U^{-1} U=V^{-1} V=\left(\left\{x, x^{-1}\right\}, 1\right)$ and $V^{-1}=\left(\left\{x^{-1}, x^{-2}\right\}, 1\right)$. Thus not only are $U$ and $V$ not free generators for $\langle K\rangle$ but $\langle K\rangle$ is not a free inverse subsemigroup of $F$.

\section{References}

[1] A.H. Clifford and G.B. Preston, The algebraic theory of semigroups (Math. Surveys 7 (I). Amer. Math. Soc., Providence, Rhode Island, 1961).

[2] Carl Eberhart and John Selden, "One parameter inverse semigroups", Trans. Amer. Math. Soc. (to appear).

[3] D.B. MCAlister, "A homomorphism theorem for semigroups", J. London Math. Soc. 43 (1968), 355-366.

[4] W.D. Munn, "A certain sublattice of the lattice of congruences on a regular semigroup", Proc. Combridge Philos. Soc. 60 (1964), 385-391.

[5] Б.M. Шайн [В.M. S̆атn], "Н теорни обобщенных групп" [On the theory of generalized groups ], Dok Z. Akad. Nauk SSSR 153 (1963), 296-299.

[6] H.E. Scheiblich, "Free inverse semigroups", (to appear).

[7] Eugene Schenkman, Group theory (Van Nostrand, Princeton, New Jersey; Toronto; New York; London; 1965).

\footnotetext{
Department of Mathematics, Simon Fraser University,

Burnaby,

British Columbia,

Canada.
} 